\title{
Comparison of anterior segment parameters and axial length measurements performed on a Scheimpflug device with biometry function and a reference optical biometer
}

\author{
Maria Muzyka-Woźniak • Adam Oleszko
}

Received: 2 November 2017/Accepted: 13 April 2018/Published online: 26 April 2018

(C) The Author(s) 2018

\begin{abstract}
Purpose To compare measurements of axial length $(\mathrm{AL})$, corneal curvature $(\mathrm{K})$, anterior chamber depth (ACD) and white-to-white (WTW) distance on a new device combining Scheimpflug camera and partial coherence interferometry (Pentacam AXL) with a reference optical biometer (IOL Master 500). To evaluate differences between IOL power calculations based on the two biometers.

Methods Ninety-seven eyes of 97 consecutive cataract or refractive lens exchange patients were examined preoperatively on IOL Master 500 and Pentacam AXL units. Comparisons between two devices were performed for AL, K, ACD and WTW. Intraocular lens (IOL) power targeting emmetropia was calculated with SRK/T and Haigis formulas on both devices and compared.

Results There were statistically significant differences between two devices for all measured parameters $(P<0.05)$, except ACD $(P=0.36)$. Corneal curvature measured with Pentacam AXL was
\end{abstract}

M. Muzyka-Woźniak · A. Oleszko ( $₫)$

Spektrum Eye Clinic, Zaolziańska 4, 53-334 Wrocław, Poland

e-mail: adam.oleszko@pwr.edu.pl

A. Oleszko

Department of Biomedical Engineering, Faculty of Fundamental Problems of Technology, Wroclaw University of Science and Technology, Wybrzeże Stanisława Wyspiańskiego 27, 50-370 Wrocław, Poland significantly flatter then with IOL Master. The mean difference in AL was clinically insignificant (0.01 mm; 95\% LoA $0.16 \mathrm{~mm}$ ). Pentacam AXL yielded higher IOL power in $75 \%$ of eyes for Haigis formula and in $62 \%$ of eyes for SRK/T formula, with a mean difference within $\pm 0.5 \mathrm{D}$ for 72 and $86 \%$ of eyes, respectively.

Conclusions There were statistically significant differences between AL, K and WTW measurements obtained with the compared biometers. Flatter corneal curvature measurements on Pentacam AXL necessitate formulas optimisation for Pentacam AXL.

Keywords Scheimpflug device - Optical biometry · IOL calculation · Axial lengths · Keratometry $\cdot$ Whiteto white distance

\section{Introduction}

Precision requirements regarding power calculation for intraocular lens (IOL) are growing. With new multifocal IOLs on board and refractive or presbyopic lens exchange becoming more popular, there is no room for inaccurate measurements. Addressing posterior corneal astigmatism has become an important issue when dealing with toric monofocal or toric multifocal IOLs [1-4]. The posterior corneal curvature can be assessed with optical coherence tomography (OCT) systems (Casia SS-1000, Tomey Corp.) and 
Scheimpflug camera-based devices such as Pentacam $\mathrm{HR}^{\circledR}$ (Oculus Optikgerate GmbH), Galilei ${ }^{\circledR}$ (Ziemer Ophthalmic Systems AG) and Sirius ${ }^{\circledR}$ (Schwind). Some of these devices, used primarily for 3-dimensional anterior segment analysis, have been recently redesigned to feature an optical biometry function. The new Galilei G6 ${ }^{\circledR}$ and Pentacam AXL ${ }^{\circledR}$ can now also be used also as biometers for IOL power calculations $[5,6]$.

There are few studies comparing these new devices to the "gold standard" optical biometer, the IOL Master 500 [7-9]. Recent study retrospectively compared the Galilei G6 with the IOL Master 500. The devices provided similar mean IOL power, axial length (AL), keratometric and anterior chamber depth (ACD) measurements, but the authors suggested they should not be used interchangeably for biometric measurements and IOL power calculations [7]. Studies comparing the Pentacam AXL to other optical biometers found inconsistent results. Shajari compared the Pentacam AXL with the IOL Master 500 and the IOL Master 700 and found no statistically significant differences in AL, keratometry and ACD measurements performed with the three devices [8]. Sel comparing the Pentacam AXL with the IOL Master 700 found significant differences for keratometry values, AL and ACD measurements [9].

The Pentacam AXL is a single rotating Scheimpflug camera device combined with optical biometry based on partial coherence interferometry (PCI) [5]. The repeatability of measurements obtained with the Pentacam HR and the Pentacam AXL was confirmed $[8,10,11]$. However, the question arises, if the Pentacam AXL can be used as a solo optical biometer in an ophthalmic setting.

The aim of our study was to compare the measurement results for AL, keratometry, ACD and white-towhite (WTW) obtained with the Pentacam AXL and the IOL Master 500.

\section{Patients and methods}

This cross-sectional study enrolled 97 eyes of 97 consecutive patients who came for preoperative measurements before cataract surgery or refractive lens exchange in a private hospital (2500 cases/year). The study was conducted in compliance with the principles of the Declaration of Helsinki and approved by local ethics committee of Lower Silesia Medical Council. Written informed consent was obtained from all study participants.

Patients with corneal or retinal diseases, including age-related macular degeneration, and previous ocular trauma were excluded. Only patients with good fixation were eligible. For each subject, we assessed only one eye, either the one with the more advanced cataract, planned to be operated first, or the phakic eye if the other was already pseudophakic. The subjects were examined prospectively on two devices: IOL Master 500 (software version 5.02.0590) and Pentacam AXL (software version 1.20r111). IOL Master 500 was considered a reference biometer. The measurements for individual subjects on the two devices were taken no more than $15 \mathrm{~min}$ apart to avoid the effects of diurnal variations in corneal indices. The IOL Master exam was always performed as first. Exams were included only if positively evaluated by automatic quality checks on both devices.

The Pentacam AXL is a rotating Scheimpflug camera combined with optical biometry based on PCI. Its light source is a blue light-emitting diode (LED) with a wavelength of $475 \mathrm{~nm}$. The rotational measuring procedure generates Scheimpflug images in three dimensions, with the dot matrix fine-meshed in the centre due to the rotation. It takes a maximum of $2 \mathrm{~s}$ to generate a complete image of the anterior eye segment. Any eye movement is detected by a second camera and corrected for in the process. The entire cornea is analysed in multiple ways. The simulated $\mathrm{K}$ (SimK) is derived from images taken exclusively from the anterior corneal surface, over a 3-mm ring [5].

IOL Master 500 is a PCI optical biometer, with a 780-nm laser diode infrared light. It measures the ACD, which corresponds to the distance between the corneal epithelium and the anterior lens surface, through a lateral slit illumination. For keratometry, it uses a hexagonal pattern of six points in a 2.3-mmdiameter zone.

Both devices use a keratometric index of 1.3375 to convert anterior corneal curvature measurements in millimetres to corneal power in dioptres (D). Pentacam AXL calculates two ACDs: internal (from corneal endothelium to the anterior lens surface) and external (from corneal epithelium to the anterior lens surface). For the purpose of this study, only external ACD was included. 
The data from the two devices taken for analysis included: axial length (AL), corneal curvature in steep meridian (Ks), corneal curvature in flat meridian (Kf), mean corneal curvature $(\mathrm{Km})$, anterior chamber depth (ACD) and white-to-white distance (WTW).

For all eyes, IOL power (SN60WF, Alcon ${ }^{\circledR}$ ) targeting emmetropia was calculated with Haigis and SRK/T formulas using the IOL Master measurements with the User Group for Laser Interference Biometry (ULIB) constants for IOL Master 500 [12-14]. The ULIB constants for Pentacam AXL have been derived from constants for IOL Master. The IOL power indicating an absolute value of target refraction closest to zero was used for analysis.

\section{Statistical analysis}

Statistical analyses were performed using MATLAB R2009b software (MathWorks, Inc.). We calculated the sample size needed to detect an AL difference of $0.02 \mathrm{~mm}$ between two devices. Fifty-seven eyes were required for a significance level $(\alpha)$ of 0.05 and a test power of 0.80 . Normality of data was assessed by the Kolmogorov-Smirnov test. All measurement data exhibited normal distribution. A paired sample $t$ test was performed to evaluate statistical significance of differences between readings from the two devices. A difference was deemed statistically significant if its $P$ value was less than 0.05 . Intraclass correlation coefficient (ICC) was calculated to assess agreement between measurements [15]. ICC values higher than 0.900 were considered as having a high degree of agreement. Additionally, Bland-Altman plots were used to evaluate the agreement between devices. The mean difference and 95\% limits of agreement (LoA) were calculated. The plots were created with Origin 8.0 software (OriginLab, Co.).

\section{Results}

Out of 97 enrolled eyes, 10 eyes of 10 patients were excluded because of persistent errors in repeated AL measurements with the Pentacam AXL, due to dense crystalline lens opacity (seven eyes) or high axial myopia (three eyes), although the respective AL measurement presented no difficulties with the IOL Master 500. The final analysis included 87 eyes of 87 subjects. Subject mean age was $58 \pm 15$ (SD) (range 27-82 years), 51 were female. The mean axial length was $23.29 \pm 1.82 \mathrm{~mm}$ (range 19.34-28.49 mm), as measured with IOL Master 500.

Table 1 shows mean values and differences with 95\% confidence interval (CI), LoA, ICC and P values for all measured parameters, comparing between IOL Master and Pentacam AXL. The paired sample t test showed statistically significant differences between IOL Master 500 and Pentacam AXL measurements for $\mathrm{AL}, \mathrm{Kf}, \mathrm{Ks}, \mathrm{Km}$ and WTW $(P<0.05)$, while no significant difference was found for ACD measurements $(P=0.36)$. The ICC values between the two devices were high and exceeding 0.900 for all analysed parameters, except WTW (ICC $=0.431$ ). The Bland-Altman plots for $\mathrm{AL}, \mathrm{Kf}, \mathrm{Ks}, \mathrm{Km}$ and WTW are shown in Figs. 1, 2, 3, 4, 5 and 6. $K$ values are reported in $\mathrm{mm}$.

The Pentacam AXL yielded lower values for AL and WTW as well as flatter corneal curvatures in flat and step meridians. The mean difference between devices in AL measurements was $0.01 \mathrm{~mm}(95 \% \mathrm{CI}$ 0.01-0.02). The difference between mean curvature radii reached significance $(P<0.05)$ with Pentacam AXL results being higher by a mean difference of $0.05 \mathrm{~mm}$ and $0.06 \mathrm{~mm}$ for the flat and the steep meridian, respectively. WTW distance measured with Pentacam AXL was significantly shorter then with IOL Master $500(11.5 \pm 0.4 \mathrm{~mm}$ and $11.9 \pm 0.5 \mathrm{~mm}$, respectively). ACD was the only parameter that showed no statistically significant difference between devices, with mean value of $-0.01 \pm 0.13$ $(P=0.36)$ and good agreement $(\mathrm{ICC}=0.945)$.

Table 2 shows the mean IOL powers (SN60WF, Alcon ${ }^{\circledR}$ ) calculated on both devices, aiming for "zero" postoperative refraction. Ten eyes were excluded from the analysis because their calculated IOL power exceeded the commercially available range. There was a statistically significant difference between IOL power selected by the two devices, with Pentacam AXL showing higher IOL power in $75 \%$ of eyes with the Haigis formula and in $62 \%$ of eyes with the SRK/T formula (Fig. 7). The differences were within $\pm 0.5 \mathrm{D}$ in 71.6 and $86.4 \%$ of eyes with Haigis and SRK/T formula, respectively (Table 2).

The distribution of IOL powers differences between devices, as calculated with two formulas (Haigis and SRK/T) to achieve emmetropia, is shown in Fig. 7. 
Table 1 Comparison of measurements obtained from IOL Master 500 and Pentacam AXL

\begin{tabular}{|c|c|c|c|c|c|c|c|}
\hline \multirow[b]{2}{*}{$\begin{array}{l}\text { Parameter } \\
(\mathrm{mm})\end{array}$} & \multirow[b]{2}{*}{$\begin{array}{l}\text { IOL Master } \\
(\text { Mean } \pm \text { SD) }\end{array}$} & \multirow[b]{2}{*}{$\begin{array}{l}\text { Pentacam } \\
(\text { Mean } \pm \text { SD) }\end{array}$} & \multirow[b]{2}{*}{$\begin{array}{l}\text { LoA } \\
95 \%\end{array}$} & \multicolumn{2}{|c|}{$\begin{array}{l}\text { Difference IOL Master 500- } \\
\text { Pentacam AXL }\end{array}$} & \multirow[b]{2}{*}{$* P$ Value } & \multirow[b]{2}{*}{ ICC } \\
\hline & & & & Mean \pm SD & $95 \%$ CI & & \\
\hline $\mathrm{AL}$ & $23.29 \pm 1.82$ & $23.27 \pm 1.83$ & 0.16 & $0.01 \pm 0.04$ & $0.01,0.02$ & $<0.001$ & 0.999 \\
\hline $\mathrm{K}$ flat & $7.78 \pm 0.29$ & $7.83 \pm 0.29$ & 0.21 & $-0.05 \pm 0.05$ & $\begin{array}{r}-0.06 \\
-0.04\end{array}$ & $<0.001$ & 0.967 \\
\hline K step & $7.59 \pm 0.31$ & $7.64 \pm 0.30$ & 0.20 & $-0.06 \pm 0.05$ & $\begin{array}{r}-0.07 \\
-0.05\end{array}$ & $<0.001$ & 0.966 \\
\hline $\mathrm{K}$ mean & $7.68 \pm 0.29$ & $7.74 \pm 0.28$ & 0.17 & $-0.05 \pm 0.04$ & $\begin{array}{r}-0.06 \\
-0.05\end{array}$ & $<0.001$ & 0.967 \\
\hline WTW & $11.9 \pm 0.5$ & $11.5 \pm 0.4$ & 0.6 & $0.4 \pm 0.2$ & $0.3,0.5$ & $<0.001$ & 0.431 \\
\hline $\mathrm{ACD}$ & $3.01 \pm 0.39$ & $3.02 \pm 0.39$ & 0.51 & $-0.01 \pm 0.13$ & $-0.04,0.02$ & 0.360 & 0.945 \\
\hline
\end{tabular}

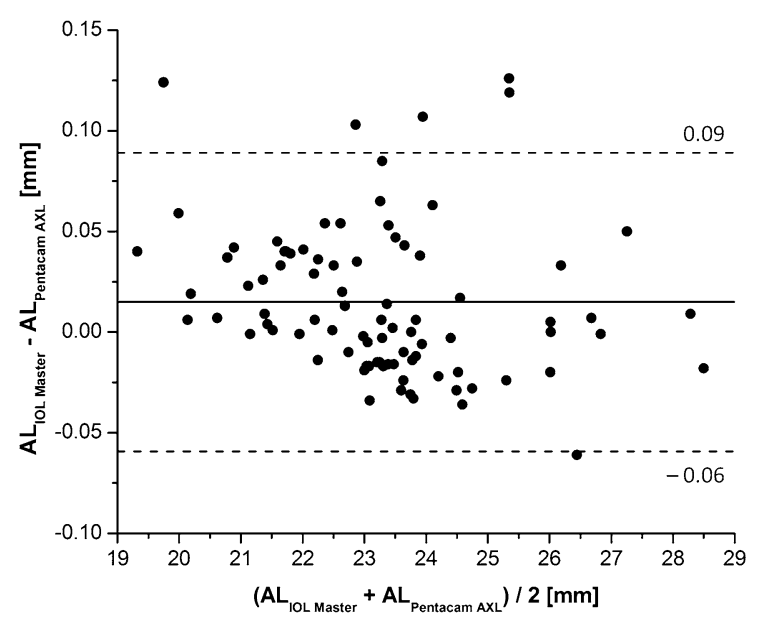

Fig. 1 Bland-Altman plots showing the agreement between IOL Master 500 and Pentacam AXL in AL measurements. The bold lines represent the mean difference between two devices. The dashed lines represent $95 \%$ limits of agreement (LoA). The percentage of points within $95 \%$ LoA is 93

\section{Discussion}

We planned the present study to establish if we can rely on the Pentacam AXL as a single optical biometer in our setting (private hospital). It seems economically questionable as well as time-consuming to use two optical biometers to examine every patient, while the option of measuring the posterior corneal curvature is a promising feature of an optical biometer. We therefore compared the new Pentacam AXL with the existing IOL Master 500, performing examinations on

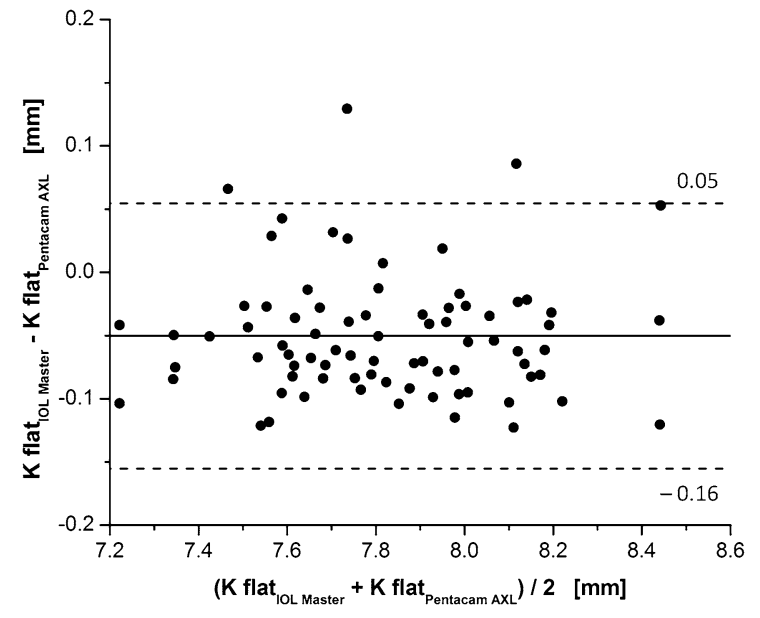

Fig. 2 Bland-Altman plots showing the agreement between IOL Master 500 and Pentacam AXL in Kf measurements. The bold lines represent the mean difference between two devices. The dashed lines represent $95 \%$ limits of agreement (LoA). The percentage of points within $95 \%$ LoA is 96

consecutive patients presenting for cataract surgery or refractive lens exchange.

We found that axial length measurements obtained with the IOL Master 500 were slightly but significantly higher $(0.01 \mathrm{~mm})$ than those obtained with the Pentacam AXL. This is close to the results of two recent studies, which report that the Pentacam AXL returns readings that, respectively, differ by a mean of $0.05 \mathrm{~mm}$ relative to the IOL Master 700 [9] and $0.026 \mathrm{~mm}$ relative to the IOL Master 500 [8]. Such a small difference should not influence IOL power calculation. 


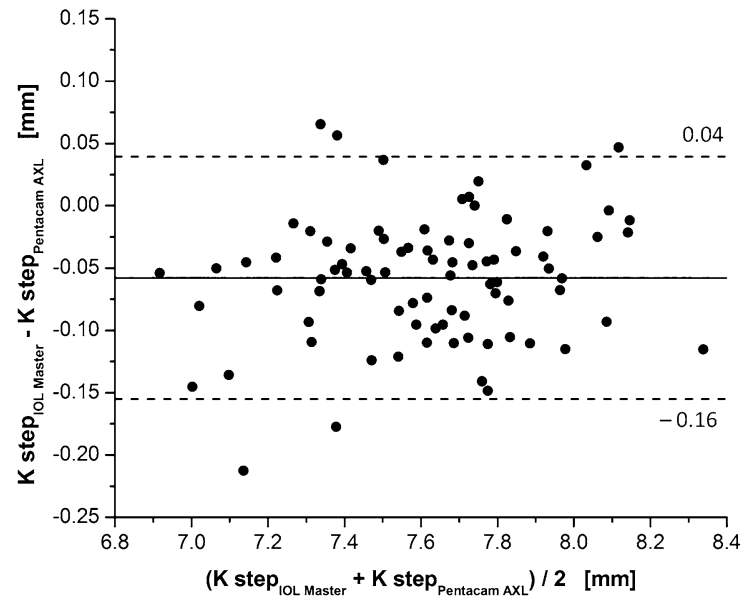

Fig. 3 Bland-Altman plots showing the agreement between IOL Master 500 and Pentacam AXL in Ks measurements. The bold lines represent the mean difference between two devices. The dashed lines represent $95 \%$ limits of agreement (LoA). The percentage of points within $95 \%$ LoA is 94

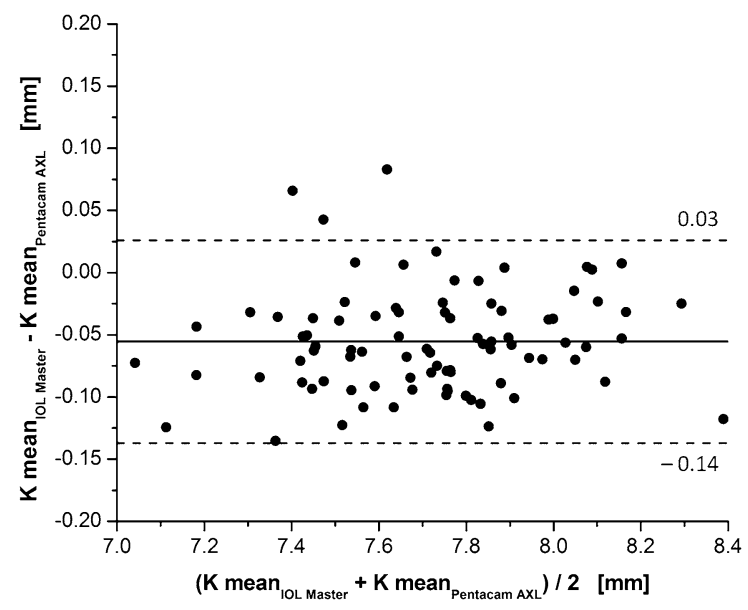

Fig. 4 Bland-Altman plots showing the agreement between IOL Master 500 and Pentacam AXL in Km measurements. The bold lines represent the mean difference between two devices. The dashed lines represent $95 \%$ limits of agreement (LoA). The percentage of points within $95 \%$ LoA is 96

Both devices use the same proven PCI biometry. Nevertheless, we were not able to measure AL with the Pentacam AXL for 10 eyes of 10 patients out of the total 97 , even though these eyes were successfully measured with the with IOL Master 500, with a wide range of AL (19.34-28.49 mm). The eyes in question featured advanced cataract or high axial myopia. New swept source OCT biometers are expected to have

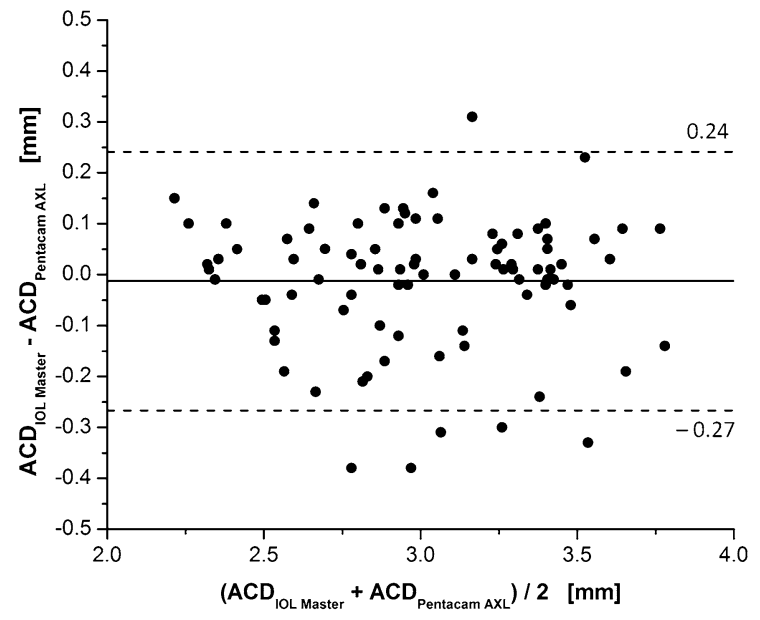

Fig. 5 Bland-Altman plots showing the agreement between IOL Master 500 and Pentacam AXL in ACD measurements. The bold lines represent the mean difference between two devices. The dashed lines represent $95 \%$ limits of agreement (LoA). The percentage of points within $95 \%$ LoA is 93

higher success rates in measuring AL in cases of dense and posterior subcapsular cataracts [16].

The agreement between Pentacam AXL and IOL Master 500 in ACD measurements was very good, with a statistically nonsignificant difference of - $0.01 \mathrm{~mm}$ (95\% CI - 0.04-0.02). An older study (2009) reported that average IOL Master ACD measurement results were $0.11 \mathrm{~mm}$ smaller than those obtained with the Pentacam. Still, it was not enough to produce clinically noticeable differences in refractive outcomes of cataract surgery [17]. It was also shown that for ACD values, the Pentacam had the smallest standard deviation when compared to IOL Master and A-scan [18].

In our study, the mean difference in corneal curvature measurements between the IOL Master 500 and the Pentacam AXL was largest for steep meridian $(-0.06 \mathrm{~mm}$, equal to $0.33 \mathrm{D}$ for a standardised keratometric refraction index of 1.3375). Another group recently found a difference of $-0.033 \mathrm{~mm}$ (equal to $0.19 \mathrm{D}$ ), also regarding the steep meridian [8]. Studies based on the former version of Pentacam or Pentacam HR also indicated differences in keratometric measurements [19-24]. Karunaratne found statistically significant differences between the mean keratometric values of the IOL Master and Pentacam equivalent keratometry readings at 2-, 3- and 4.5-mm zone measurements. The authors concluded that after constant optimisation, keratometry from the IOL 
Fig. 6 Bland-Altman plots showing the agreement between IOL Master 500 and Pentacam AXL in WTW measurements. The bold lines represent the mean difference between two devices. The dashed lines represent $95 \%$ limits of agreement (LoA). The percentage of points within $95 \%$ LoA is 95

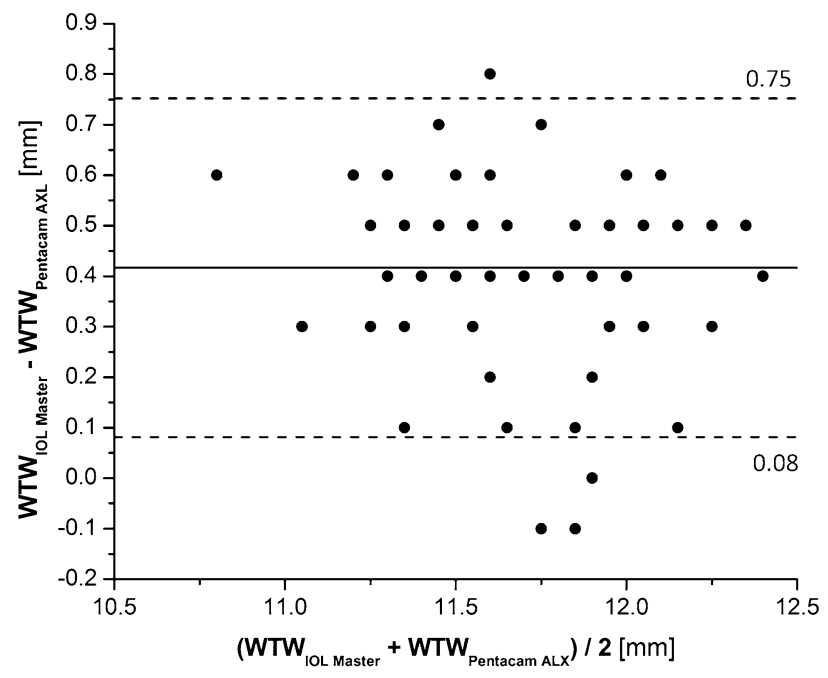

Table 2 Comparison of Haigis and SRK/T IOL power calculations (SN60WF, Alcon ${ }^{\circledR}$ ) with IOL Master 500 and Pentacam AXL data

\begin{tabular}{|c|c|c|c|c|c|c|}
\hline \multirow[b]{3}{*}{ Formula } & & \multicolumn{4}{|l|}{ IOL power (D) } & \multirow[b]{3}{*}{$P$ value } \\
\hline & & \multicolumn{2}{|l|}{ Mean \pm SD } & \multicolumn{2}{|c|}{ Agreement between devices ( $\%$ of eyes) } & \\
\hline & & IOL Master 500 & Pentacam AXL & $\pm 0.50 \mathrm{D}(\%)$ & $\pm 1.00 \mathrm{D}(\%)$ & \\
\hline \multirow[t]{2}{*}{ Haigis } & Mean $\pm \mathrm{SD}$ & $21.06 \pm 4.94$ & $21.58 \pm 4.90$ & 71.6 & 95.1 & $<0.001$ \\
\hline & Range & $6.50 ; 29.50$ & $7.50 ; 29.50$ & & & \\
\hline \multirow[t]{2}{*}{$\mathrm{SRK} / \mathrm{T}$} & Mean $\pm \mathrm{SD}$ & $20.98 \pm 4.82$ & $21.34 \pm 4.78$ & 86.4 & 97.5 & $<0.001$ \\
\hline & Range & $6.50 ; 28.50$ & $7.00 ; 29.00$ & & & \\
\hline
\end{tabular}

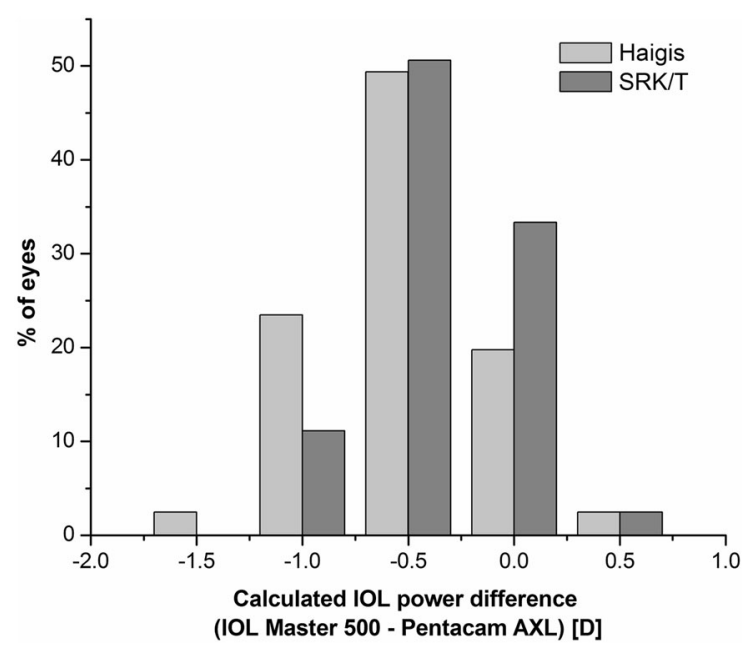

Fig. 7 Distribution of IOL power differences between IOL Master 500 and Pentacam AXL for two calculation formulas (Haigis and SRK/T) to reach emmetropia
Master and the Pentacam equivalent $4.5-\mathrm{mm}$ zone keratometry reading may be similarly effective when used in IOL power calculation formulas [22].

The Pentacam AXL measures posterior curvature of the cornea and corneal thickness, yielding total corneal power and true net power information. We provide no comparisons of the devices in this respect, as it is well known, that the cited parameters and anterior curvature readings are not comparable $[21,25]$ and cannot be used for most of the present IOL calculation formulas [26].

For calculation of IOL toricity, the Pentacam AXL offers meridional analysis method, which uses total corneal power in a $3-\mathrm{mm}$ zone $[27,28]$. For the spherical equivalent power, anterior corneal curvature values are applied. We did not analyse calculations for toric IOLs in our subjects. It is worth mentioning that the Pentacam's repeatability of corneal curvature 
readings was found to be better than that of the IOL Master 500 or IOL Master 700 [8].

It was interesting to find that WTW measurements were significantly lower on the Pentacam AXL in comparison to the IOL Master 500 with poor agreement (ICC $=0.470$ ) between the devices. A statistically significantly lower WTW distance compared to the IOL Master was also found with the Lenstar ${ }^{\circledR}$ (Haag Streit, USA) in a meta-analysis published in 2017 [29]. Another study reported a lower average WTW value on a Scheimpflug/Placido topography system as compared to the IOL Master [30]. Moreover, the IOL Master constantly yielded values exceeding measurements based on direct eye visualisation [31]. These differences must be considered in IOL formulas incorporating WTW data and in calculating phakic IOLs.

Since the ACD measurements were not statistically different and differences in $\mathrm{AL}$ were clinically insignificant, the tendency of the Pentacam AXL to provide higher calculated IOL power in our cohort can likely be explained by its flatter keratometric measurements. Compared to the IOL Master, the Pentacam AXL indicated higher IOL power, according to Haigis and SRK/T, for 75 and $62 \%$ of eyes, respectively. The ULIB SN60WF constants for the Pentacam are the same as for IOL Master 500 (A SRKT = 119; $\mathrm{a} 0=-0.769 ; \mathrm{a} 1=0.234 ; \mathrm{a} 2=0.217$ ). Our results showed that these constants are obviously not optimal for the Pentacam AXL, and all users should start with a period of "sham" measurements, which will form a basis for the needed corrections. Our cohort was not suitable for constants optimisation, because in reality we implanted our subjects with different types of IOLs. Nevertheless, our theoretical comparison indicates that optimisation of constants for the Pentacam $\mathrm{AXL}$ is a must. This is a time-consuming process, and new Pentacam AXL users should be aware of this burden.

\section{Conclusions}

The measurements results required for IOL power calculation performed on the compared devices were found to be significantly different. Pentacam AXL and IOL Master 500 should not be used interchangeably. Due to its constantly flatter corneal curvature measurements, the Pentacam AXL requires optimisation of constants used in formulas.

\section{Compliance with ethical standards}

Conflict of interest The authors declare that they have no conflicts of interest.

Informed consent Informed consent was obtained from all study subjects.

Open Access This article is distributed under the terms of the Creative Commons Attribution 4.0 International License (http:// creativecommons.org/licenses/by/4.0/), which permits unrestricted use, distribution, and reproduction in any medium, provided you give appropriate credit to the original author(s) and the source, provide a link to the Creative Commons license, and indicate if changes were made.

\section{References}

1. Reitblat O, Levy A, Kleinmann G et al (2016) Effect of posterior corneal astigmatism on power calculation and alignment of toric intraocular lenses: comparison of methodologies. J Cataract Refract Surg 42:217-225

2. Koch DD, Jenkins RB, Weikert MP et al (2013) Correcting astigmatism with toric intraocular lenses, effect of posterior corneal astigmatism. J Cataract Refract Surg 39:1803-1809

3. Koch DD (2015) The posterior cornea: hiding in plain sight. Ophthalmology 122:1070-1071

4. Hayashi K, Manabe S, Yoshida M, Hayashi H (2010) Effect of astigmatism on visual acuity in eyes with a diffractive multifocal intraocular lens. J Cataract Refract Surg 36:1323-1329

5. Pentacam User Guide. System for measuring and analysing the front part of the eye. User Guide Pentacam ${ }^{\circledR} /$ Pentacam ${ }^{\circledR}$ HR/Pentacam ${ }^{\circledR}$ AXL. https://www.pentacam.com/ fileadmin/user_upload/pentacam.de/downloads/ interpretations-leitfaden/interpretation_guideline_3rd_ edition_0915.pdf. Accessed 25 Apr 2018

6. Galilei G6 Lens Professional, Product Brochure. https:// www.galilei.ziemergroup.com/galilei-g6.html. Accessed 25 Apr 2018

7. Ventura BV, Ventura MC, Wang L, Koch DD, Weikert MP (2017) Comparison of biometry and intraocular lens power calculation performed by a new optical biometry device and a reference biometer. J Cataract Refract Surg 43:74-79

8. Shajari M, Cremonese C, Petermann K, Singh P, Müller M, Kohnen T (2017) Comparison of axial length, corneal curvature, and anterior chamber depth measurements of 2 recently Introduced devices to a known biometer. Am J Ophthalmol 178:58-64

9. Sel S, Stange J, Kaiser D, Kiraly L (2017) Repeatability and agreement of Scheimpflug-based and swept-source optical biometry measurements. Cont Lens Anterior Eye. https:// doi.org/10.1016/j.clae.2017.03.007

10. Németh G, Hassan Z, Módis L, Szalai E, Katona K, Berta A (2011) Comparison of anterior chamber depth 
measurements conducted with Pentacam $\mathrm{HR}^{\circledR}$ and IOLMaster $^{\circledR}$. Ophthalmic Surg Lasers Imaging 42:144-147

11. Rozema JJ, Wouters K, Mathysen DGP, Tassignon MJ (2014) Overview of the repeatability, reproducibility, and agreement of biometry values provided by various ophthalmic devices. Am J Ophthalmol 158:1111-1120

12. Retzlaff JA, Sanders DR, Kraff MC (1990) Development of the SRK/T intraocular lens implant power calculation formula. J Cataract Refract Surg 16:333-340 (erratum, 528)

13. Haigis W, Lege B, Miller N, Schneider B (2000) Comparison of immersion ultrasound biometry and partial coherence interferometry for intraocular lens calculation according to Haigis. Graefes Arch Clin Exp Ophthalmol 238:765-773

14. User Group for Laser Interference Biometry. http://www. ocusoft.de/ulib/. Accessed 25 Apr 2018

15. Weir JP (2005) Quantifying test-retest reliability using the intraclass correlation coefficient and the SEM. J Strength Condition Res 19:231-240

16. Kurian M, Negalur N, Das S, Puttaiah NK, Haria D, TS J, Thakkar MM (2016) Biometry with a new swept-source optical coherence tomography biometer: repeatability and agreement with an optical low-coherence reflectometry device. J Cataract Refract Surg 42:577-581

17. Utine CA, Altin F, Cakir H, Perente I (2009) Comparison of anterior chamber depth measurements taken with the Pentacam, Orbscan IIz and IOL Master in myopic and emmetropic eyes. Acta Ophthalmol 87:386-391

18. Su PF, Lo AY, Hu CY, Chang SW (2008) Anterior chamber depth measurement in phakic and pseudophakic eyes. Optom Vis Sci 85:1193-1200

19. Özyol P, Özyol E (2016) Agreement between swept-source optical biometry and Scheimpflug-based topography measurements of anterior segment parameters. Am J Ophthalmol 169:73-78

20. Reuland MS, Reuland AJ, Mishi Y, Auffarth GU (2007) Corneal radii and anterior chamber depth measurements using the IOLmaster versus the Pentacam. J Refract Surg 23:368-373

21. Saad E, Shammas MC, Shammas HJ (2013) Scheimpflug corneal power measurements for intraocular lens power calculation in cataract surgery. Am J Ophthalmol 156:460-467

22. Karunaratne N (2013) Comparison of the Pentacam equivalent keratometry reading and IOL Master keratometry measurement in intraocular power calculations. Clin Exp Ophthalmol 41:825-834

23. Hamer CA, Buckhurst H, Purslow C, Shum GL, Habib NE, Buckhurst PJ (2016) Comparison of reliability and repeatability of corneal curvature assessment with six keratometers. Clin Exp Optom 99:583-589

24. Symes RJ, Ursell PG (2011) Automated keratometry in routine cataract surgery: comparison of Scheimpflug and conventional values. J Cataract Refract Surg 37:295-301

25. Koch DD, Ali SF, Weikert MP, Shirayama M, Jenkins R, Wang L (2012) Contribution of posterior corneal astigmatism to total corneal astigmatism. J Cataract Refract Surg 38:2080-2087

26. Olsen T, Hoffmann P (2014) C constant: new concept for ray tracing-assisted intraocular lens power calculation. J Cataract Refract Surg 40:764-773

27. Fam HB, Lim KL (2007) Meridional analysis for calculating the expected spherocylindrical refraction in eyes with toric intraocular lenses. J Cataract Refract Surg 33:2072-2076

28. Savini G, Naeser K (2015) An analysis of the factor influencing the residual refractive astigmatism after cataract surgery with toric intraocular lenses. Investig Ophthalmol Vis Sci 56:827-835

29. Huang J, McAlinden C, Huang Y et al (2017) Meta-analysis of optical low-coherence reflectometry versus partial coherence interferometry biometry. Sci Rep 7:43414. https://doi.org/10.1038/srep43414

30. Huang J, Liao N, Savini G et al (2014) Comparison of anterior segment measurements with Scheimpflug, placido photography-based topography system and IOLMaster partial coherence interferometry in patients with cataracts. J Ophthalmol. https://doi.org/10.1155/2014/540760

31. Wilczyński M, Pośpiech-Zabierek A (2015) Evaluation of white-to-white distance and anterior chamber depth measurements using the IOL Master, slit-lamp adapted optical coherence tomography and digital photographs in phakic eyes. Klin Oczna 117:153-159 